\title{
Convective and Diffusive Energetic Particles Losses Induced by Shear Alfvén Waves in the ASDEX Upgrade Tokamak
}

\author{
M. García-Muñoz ${ }^{(1)},{ }^{*}$ N. Hicks ${ }^{(1)}$, R. van Voornveld ${ }^{(2)}$, I.G.J. Classen ${ }^{(1,2)}$, R.

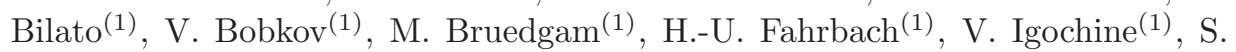 \\ Jaemsae $^{(3)}$, M. Maraschek ${ }^{(1)}$, K. Sassenberg ${ }^{(1)}$, and the ASDEX Upgrade Team \\ (1) Max-Planck-Institut für Plasmaphysik, EURATOM Association \\ Boltzmannstr. 2, D-85748 Garching, Germany \\ ${ }^{(2)}$ FOM-Institute for Plasma Physics Rijnhuizen, Association EURATOM-FOM, \\ PO Box 1207, 3430 BE Nieuwegein, The Netherlands and \\ (3) Helsinki Univ. of Technology, Association Euratom-Tekes, P.O.Box 4100, FIN-02015 HUT, Finland
}

(Dated: March 10, 2010)

\begin{abstract}
We present here the first phase-space characterization of convective and diffusive energetic particle losses induced by shear Alfvén waves in a magnetically confined fusion plasma. While single Toroidal Alfvén Eigenmodes (TAEs) and Alfvén Cascades (ACs) eject resonant fast-ions in a convective process, an overlapping of AC and TAE spatial structures leads to a large fast-ion diffusion and loss. Diffusive fast-ion losses have been observed with a single TAE above a certain threshold in the fluctuation amplitude.
\end{abstract}

PACS numbers: 52.55.-s, 52.55.Pi, 96.50.Ci, 94.05.Sd

Alfvén waves are ubiquitous in astrophysical as well as in laboratory plasmas [1]. Their interplay with energetic ions is of crucial importance to understand the energy and particle exchange in astrophysical plasmas [2] as well as to obtain a viable energy source in magnetically confined fusion devices such as ITER [3]. In astrophysics, alfvenic instabilities are thought to be responsible for the anomalous transport of particles and energy in regimes such as the heating of the solar corona or the generation of the solar wind [4-10]. In magnetically confined fusion plasmas, the excitation of shear Alfvén waves such as Reverse Shear Alfvén Eigenmodes (RSAEs) [11, 12], so called Alfvén Cascades (ACs), and Toroidal Alfvén Eigenmodes (TAEs) $[13,14]$ is of special importance for addressing the fast-ion transport across the magnetic field lines because of their potential to eject fast-ions before their thermalization $[15,16]$.

The non-linear evolution of alfvenic instabilities driven by energetic particles and the subsequent redistribution of those particles has been the focus of exhaustive theoretical studies [17-22]. A wave-particle exchange of momentum and energy takes place in tokamaks if the resonant condition $\Omega_{n, p}=n \omega_{\phi}-p \omega_{\theta}-\omega \approx 0$ is fulfilled [23]. Here, $n$ is the toroidal mode number, $p$ is the poloidal harmonic, $\omega_{\phi}$ the fast-ion precession frequency, $\omega_{\theta}$ the fast-ion poloidal frequency, $\omega$ the mode frequency and $\Omega_{n, p}$ the resonance width $[24,25]$. At each resonance, the wave particle momentum exchange, proportional to the fluctuation amplitude $\delta B$, corresponds to a radial drift of the fast-ions. This linear momentum exchange may cause convective losses of fast-ions if the right waveparticle relative phase is given. However, depending on whether modes spatial structures and phase-space reso-

*Electronic address: Manuel.Garcia-Munoz@ipp.mpg.de nances overlap local or global redistribution of fast-ions may occur [26]. If most of the relevant phase-space is covered by overlapping resonances particles can be lost via stochastic diffusion [27]. For single modes, stochastic losses caused by the overlapping of side-band resonances are proportional to $(\delta B)^{2}[18]$. Experimentally, a detailed knowledge of the wave-particle interaction can be gained from direct measurements of MHD induced fast-ion losses in fusion plasmas [28, 29]. Fast-ion loss detectors (FILD) in fusion devices obtain typically a crucial information on the phase-space of the lost ions [30-34].

In this letter, we present the phase-space characterization of convective and diffusive fast-ion losses induced by shear Alfvén waves in a magnetically confined fusion plasma. The experiments discussed here have been performed in plasmas with toroidal plasma current $I_{p} \approx$ $0.8 \mathrm{MA}$, toroidal field $B_{t}=2.0 \mathrm{~T}$, safety factor at the edge $q_{95} \approx 4.0$ and Ion Cyclotron Resonance Heating (ICRH) as main heating and fast particle source. 4.5 MW of on axis ICRF hydrogen minority heating was applied to a deuterium plasma $\left(n_{H} / n_{D} \approx 5 \%\right.$ ). Fig.1(a) shows the core line integrated electron density, $n_{e}$, and neutron rate for the discharge presented here, \#23824. Fig.1(b) and Fig.1(c) show, respectively, the Fourier spectrogram for a magnetic fluctuation signal and for a SXR signal, corresponding to a line of sight passing through the plasma core. Several coherent MHD fluctuations are visible around $110 \mathrm{kHz}$ up to $170 \mathrm{kHz}$. They correspond to TAEs with different toroidal mode numbers [35] $n$ 's $(n=3,4,5)$, as obtained from Mirnov coils [36], whose identification is confirmed also by comparison with ideal MHD calculations carried out with the CASTOR code [37]. Magnetic fluctuations chirping up in frequency from $\approx 50 \mathrm{kHz}$ up to the TAE frequencies, $f_{T A E}=V_{A} / 4 \pi q R_{0}$, are barely visible in the magnetics spectrogram. Here, $V_{A}$ is the Alfvén speed and $R_{0}$ is the major radius. These frequency chirping fluctuations has been identified as ACs 


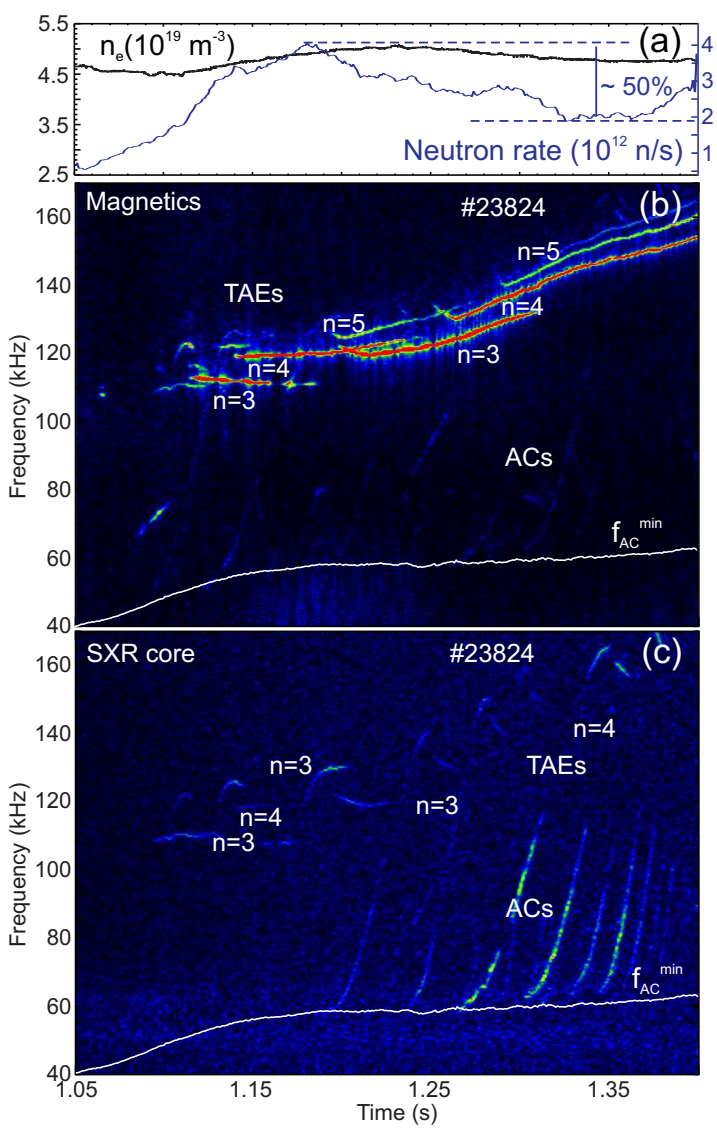

FIG. 1: AUG discharge \#23824: (a) core line integrated electron density, $n_{e}$, and neutron rate. (b) Spectrogram of an invessel magnetic pickup coil. (c) Spectrogram of a core SXR channel, the lowest AC frequency, $f_{A C}^{\min }$, is superimposed in white.

by means of the SXR emission from the plasma core as Fig.1(c) shows. The lowest AC frequency, $f_{A C}^{\min }$, in Fig.1 is mainly given by the geodesic compressibility and the toroidal coupling to the acoustic waves, as expected by the theory [38]. Pressure effects modify the local dispersion relation for shear Alfvén waves in low- $\beta$ plasmas: $\omega_{A C} \approx\left|\frac{m}{q_{\min }-n}\right| \cdot \frac{V_{A}}{R_{0}}$, with $\mathrm{m}$ the poloidal mode number. The modified minimum of the local dispersion relation is then given by

$$
\omega_{A C}^{\min } \approx \frac{\sqrt{2}}{R_{0}} \cdot C_{s}=\frac{\sqrt{2}}{R_{0}} \cdot\left(\frac{T_{i}}{m_{i}}\right)^{1 / 2} \cdot\left(\frac{7}{4}+\frac{T_{e}}{T_{i}}\right)^{1 / 2} .
$$

An excellent agreement between the experimental lowest $\mathrm{AC}$ frequency and the modified dispersion relation given in Eq.1 is clearly visible. The estimated $f_{A C}^{\min }$ has been calculated assuming $T_{e}=T_{i}$ and taking $T_{e}$ from an Electron Cyclotron Emission (ECE) radiometer channel near the AC localization. The neutron rate in Fig.1(a) shows a drop of $\approx 50 \%$ in the presence of strong TAEs and ACs, indicating a redistribution of fast deuterium ions created by high harmonic ICRH.

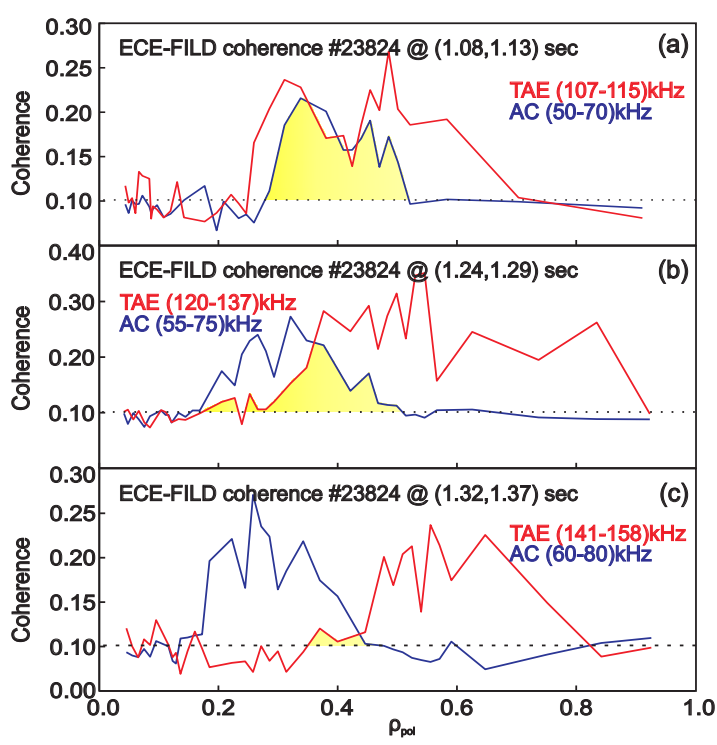

FIG. 2: AUG discharge \#23824: ACs and TAEs radial structures obtained by means of ECE-FILD cross-correlation. AC radial structures are reconstructed at the AC lowest frequency. Different time intervals are displayed; (a) for (1.081.13) sec, (b) for (1.24-1.29) sec and (c) for $(1.32,1.37)$ sec. The yellow areas highlight the regions with overlapping TAEAC internal structures well before the AC-TAE transition.

Fluctuations in the electron temperature profile caused by the ACs and TAEs [39] have been measured with the ECE radiometer at high resolution in space $(\approx 1 \mathrm{~cm})$ and time (1 MHz sampling rate). The $\mathrm{AC}$ and TAE radial structures have been reconstructed by means of ECEFILD cross-correlation techniques. Fig.2 shows the normalized cross power spectral densities (coherence) as a function of $\rho_{\text {pol }}$ and frequency for $50 \mathrm{~ms}$ time intervals. The AC and TAE radial structures were obtained by selecting and averaging a certain frequency band of the coherence. The selected AC and TAE frequency bands are given in Fig.2. Fig.2(a) shows global TAEs extended from $\rho_{\text {pol }} \approx 0.23$ up to the edge with broad ACs localized at $\rho_{\text {pol }} \approx 0.4$. A complete overlapping of AC-TAE radial structures is clearly visible. Fig.2(b) shows the AC-TAE radial structures for a later time interval. TAEs shift outwards becoming more localized while ACs shift inwards becoming also more localized at $\rho_{\text {pol }} \approx 0.3$. The overlapping region (highlighted in yellow) becomes smaller. Finally, Fig.2(c) shows a neglectable AC-TAE spatial overlapping with ACs and TAEs well localized at $\rho_{\text {pol }} \approx 0.3$ and $\rho_{\text {pol }} \approx 0.6$ respectively. The largest ACs and TAEs presented here caused a normalized $T_{e}$ perturbation of $\delta T_{e} / T_{e} \approx 0.009$.

In order to fully characterize the orbits of the lost ions and identify the wave-particle resonances responsible for the losses, the phase-space (energy and pitch angle) of the fast-ion losses is shown in Fig.3. In the presence of multiple AEs, e.g. $t=1.36 \mathrm{~s}$, fast-ions are ejected within a broad energy range with gyroradius from $\approx 35 \mathrm{~mm}$ up to $\approx 105 \mathrm{~mm}$, see Fig.3(a). For the magnetic field at the 

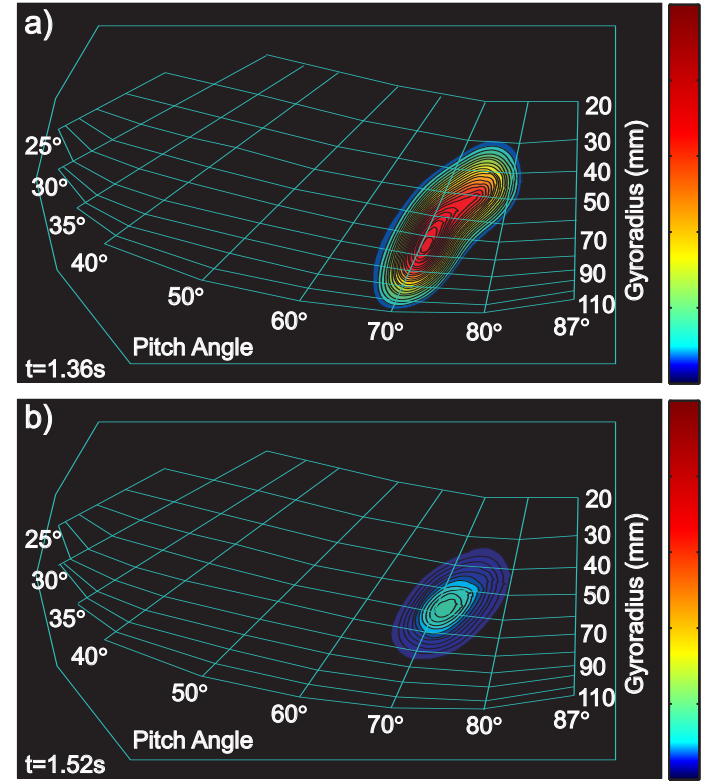

FIG. 3: AUG discharge \#23824: CCD frames showing the light patterns produced by ACs and TAEs induced fast-ion losses in particle phase-space. (a) Fast-ion losses at $\mathrm{t}=1.36 \mathrm{~s}$. (b) Fast-ion losses at $\mathrm{t}=1.52 \mathrm{~s}$.

probe, $\approx 1.6 T$, this gyroradii range corresponds to hydrogen ions with energies between $\approx 0.2 \mathrm{MeV}$ and $\approx 1.4$ $\mathrm{MeV}$. As expected from ICRF heated plasmas the fastion losses appear at high pitch angles between $67^{\circ}$ and $80^{\circ}$. The phase-space of lost fast-ions changes strongly during the evolution of the AE activity, showing a completely different pattern within the next 200 ms. Fig.3(b) presents fast-ion losses well localized at high pitch angles $\left(\approx 71^{\circ}\right)$ and energies (gyroradius $\approx 60 \mathrm{~mm}$ ).

A Fourier analysis of the fast-ion loss signal allows to identify the MHD fluctuations responsible for these losses. Fig.4(a) shows this exercise. The AC-TAE details of the FILD spectrogram are striking. A clear correlation between the TAE frequency pattern and the fast-ion loss frequencies is observed in both magnetics, Fig.1(b), and FILD, Fig.4(a), spectrograms. Although all TAEs eject resonant ions, the relative amplitude of the measured losses with respect to the fluctuation amplitude does not depend only on the magnetic fluctuation amplitude e.g. the losses measured at the $n=4$ TAE frequency are not as strong as one could expect from its large fluctuation amplitude in Fig.1(b). Changes in the spatial distribution of the losses could explain this observation as the measurements are performed at a single poloidal position. In addition, fast-ion losses chirping in frequency from approximately the geodesic frequency, $f_{A C}^{\min }$, up to the TAE frequency, $f_{T A E}$, emerge following the typical $\mathrm{AC}$ frequency pattern. The $\mathrm{AC}$ induced resonant losses appear stronger ( $\approx 10 \%$ of the total resonant losses) in the frequency range where the ACs interact with the Alfvén-acoustic branch near the $f_{A C}^{m i n}$ and during the ACTAE transition.

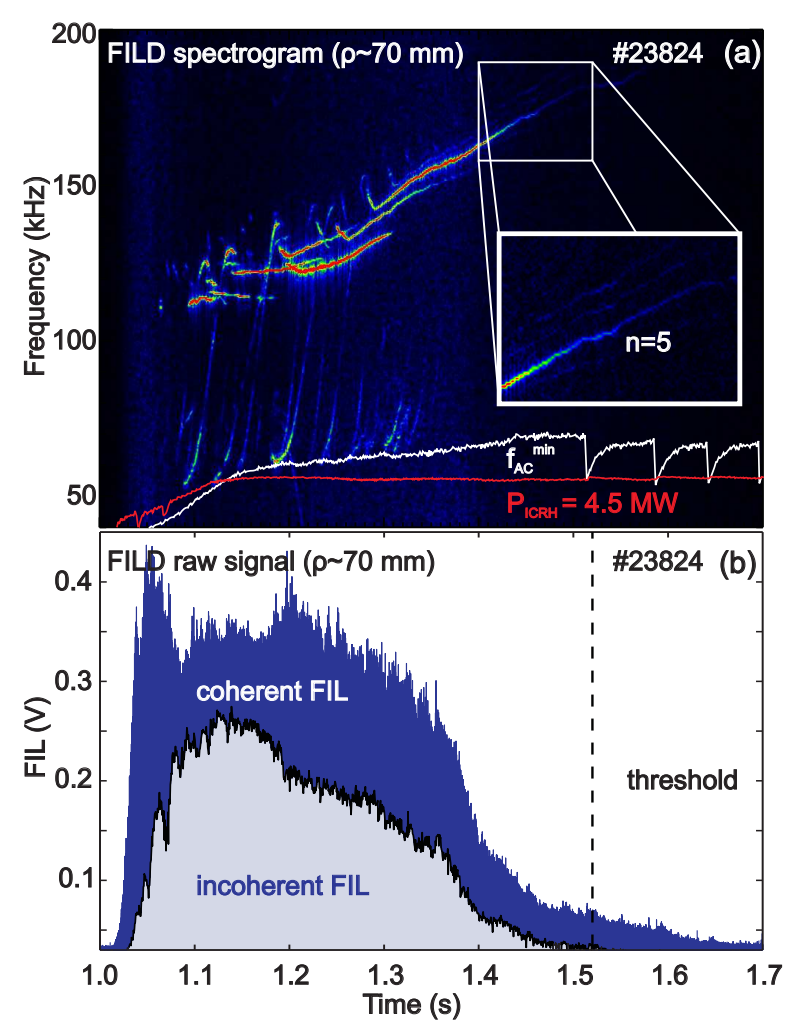

FIG. 4: AUG discharge \#23824: (a) spectrogram of the fastion loss signal with gyroradius $\approx 70 \mathrm{~mm}$. The lowest AC frequency, $f_{A C}^{\min }$, is superimposed in white while the total ICRH power $\left(\mathrm{P}_{I C R H}\right)$ is superimposed in red. The insert shows the single TAE responsible for the onset of the incoherent losses. (b) Fast-ion loss signal. The coherent and incoherent components of the losses are highlighted. The vertical dashed line depicts the threshold for the incoherent losses.

The raw data of the Fourier-analyzed fast-ion loss signal shown in Fig.4(a) is presented in Fig.4(b) to investigate the diffusive and convective character of the losses. The signal consists of a modulated (coherent) signal sitting on an incoherent background whose amplitude varies with time. The coherent component of the fast-ion losses is correlated in frequency and phase with the corresponding magnetic fluctuation, giving rise to the spectrogram shown in Fig.4(a). The incoherent component is dominant, up to $80 \%$ of the total losses, in the presence of multiple frequency chirping AEs, $\mathrm{t} \approx(1.1-1.3) \mathrm{s}$, and decreases when the number of modes decreases. However it should be noted that it is not zero when only one mode is ejecting ions with a relatively large amplitude, $\mathrm{t} \approx(1.42-1.52) \mathrm{s}$, as discussed later. During the time window $\mathrm{t} \approx(1.52-1.7)$ s only coherent losses induced by a single TAE, $\mathrm{n}=5$, are visible. Going backwards in time, the incoherent losses of ions with gyroradius $\approx 60-70$ $\mathrm{mm}$ appear, for $\mathrm{t} \leq 1.52 \mathrm{~s}$, when the local maximum radial displacement of the magnetic field lines is larger than $\approx$ $2 \mathrm{~mm}$ as measured by its fluctuation induced on the SXR emission. This threshold in the fluctuation amplitude is depicted in Fig.4(b) with a vertical dashed line. The ba- 

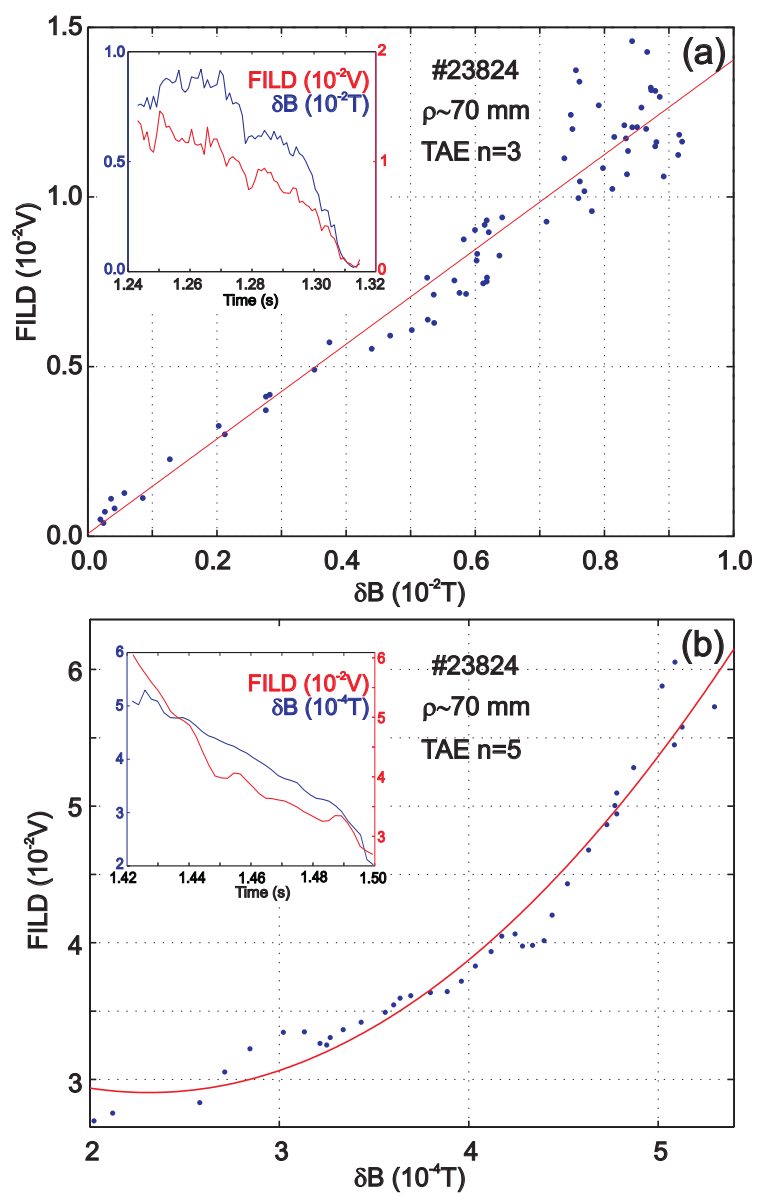

FIG. 5: AUG discharge \#23824: Fast-ion loss rate. (a) Linear dependence of the coherent losses at the TAE $n=3$ frequency on the MHD fluctuation amplitude. (b) Quadratic dependence of the incoherent losses on the TAE $n=5$ fluctuation amplitude.

sic properties of the coherent and incoherent losses are investigated through their dependence on the magnetic fluctuation amplitude. Tracking the frequencies of the individual fluctuations in both, magnetics and FILD spectrograms, we get the relationship between the coherent fast-ion losses and the corresponding magnetic fluctuation amplitude. Fig.5(a) shows this exercise for the TAE $\mathrm{n}=3$ between $\mathrm{t}=1.24 \mathrm{~s}$ and $\mathrm{t}=1.32 \mathrm{~s}$. A clear linear depen- dence is visible during the whole time window, showing the convective character of the underlying loss mechanism. A similar analysis has been done for the incoherent losses shown in Fig.4(b). The envelope of the incoherent losses, black curve in Fig.4(b), is plotted in Fig.5(b) as a function of the amplitude of the TAE $n=5$ for a time interval close to the onset of the incoherent losses, insert in Fig.5(b), $\mathrm{t}=(1.42,1.50) \mathrm{s}$. A clear quadratic dependence has been obtained, strongly suggesting a diffusive mechanism involving several resonances in phase-space. This is supported by the data shown in Fig.3(a) which indicates that the losses cover a larger domain in phase-space when the incoherent signal is not zero. It should also be underlined that, as shown in Fig.4(b), the incoherent component is even larger in the presence of several modes, which is also expected to induce a diffusive fast-ion transport.

In summary, we have characterized the phase-space of convective and diffusive energetic particle losses induced by shear Alfven waves in a magnetically confined fusion plasma. Time-resolved energy and pitch angle measurements of fast-ion losses correlated in frequency and phase with ACs and TAEs have allowed to identify both loss mechanisms. Single ACs and TAEs eject resonant fastions in a convective process directly proportional to the fluctuation amplitude, $\delta B$. The overlapping of multiple $\mathrm{AC}$ and TAE spatial structures leads to a large diffusive loss. Since these structures propagate in the plasma with different phase velocities, a simple explanation in terms of stochastisation in real space can not be given. It remains a subject of further studies to explain how incoherent losses can occur under these conditions. For single TAEs, diffusive losses of fast-ions, scaling as $(\delta B)^{2}$, are observed for local radial displacements of the magnetic field lines larger than $\approx 2 \mathrm{~mm}$. The results presented here may be of general interest for better understanding the wave-particle interactions and subsequent energy and particle transport in fusion devices as well as in astrophysical plasmas.

\section{Acknowledgments}

The authors wish to thank H. L. Berk (The University of Texas at Austin), F. Ryter and H. Zohm for very fruitful discussions.
[1] ALFVÉN, H., Nature 150 (1942) 405.

[2] MARSCH, E., Living Rev. Solar Phys. 3 (2006) 1.

[3] SHIMADA, M. et al., Nucl. Fusion 47 (2007) S1.

[4] PARKER, E. N., ApJ 372 (1991) 719.

[5] MILlER, J. A. and ROBERTS, D. A., ApJ. 452 (1995) 912.

[6] CRANMER, S. R. and VAN BALLEGOOIJEN, A. A., ApJ 594 (2003) 573.

[7] ERDELYI, R. and FEDUN, V., Science 318 (2007) 1572.

[8] DE-PONTIE, B. et al., Science 318 (2007) 1574.
[9] CIRTAIN, J. W. et al., Science 318 (2007) 1580.

[10] ARANEDA, J. A. et al., Phys. Rev. Lett. 102 (2009) 175001.

[11] BERK, H. L. et al., Phys. Rev. Lett. 87 (2001) 185002.

[12] SHARAPOV, S. E. et al., Phys. Lett. A 289 (2001) 127.

[13] WONG, K. L. et al., Phys. Rev. Lett. 66 (1991) 1874.

[14] HEIDBRINK, W. W., Nucl. Fusion 31 (1991) 1635.

[15] HEIDBRINK, W. W. et al., Phys. Rev. Lett. 99 (2007) 245002.

[16] FASOLI, A. et al., Nucl. Fusion 47 (2007) S264. 
[17] BERK, H. L. et al., Phys. Rev. Lett. 68 (1992) 3563.

[18] SIGMAR, D. et al., Phys. Fluids B 4 (1992) 1506.

[19] MYNICK, H. E., Phys. Fluids B 5 (1993) 1471.

[20] BREIZMAN, B. N. et al., Phys. Fluids B 5 (1993) 3217.

[21] TODO, Y. et al., Phys. Plasmas 10 (2003) 2888.

[22] VLAD, G. et al., Nucl. Fusion 49 (2009) 075024.

[23] WHITE, R. B. et al., Phys. Fluids 26 (1983) 2958.

[24] BERK, H. L. et al., Nucl. Fusion 35 (1995) 1661.

[25] PINCHES, S. D. et al., Nucl. Fusion 46 (2006) S904.

[26] BERK, H. L. et al., Phys. Plasmas 2 (1995) 3007.

[27] CHIRIKOV, B. V., Phys. Reports 52 (1979) 263.

[28] HEIDBRINK, W. W. et al., Phys. Rev. Lett. 53 (1984) 1905.

[29] NAGAOKA, K. et al., Phys. Rev. Lett. 100 (2008) 065005.

[30] ZWEBEN, S. J. et al., Nucl. Fusion 39 (1999) 1097.
[31] ISOBE, M. et al., Nucl. Fusion 46 (2006) S918.

[32] DARROW, D. et al., Nucl. Fusion 48 (2008) 084004 (6pp).

[33] GARCIA-MUNOZ, M. et al., Phys. Rev. Lett. 100 (2008) 055005

[34] GARCIA-MUNOZ, M. et al., Rev. Sci. Instrum. 80 (2009) 053503.

[35] WESSON, J. A., Tokamaks, Clarendon Press, Oxford, 1997.

[36] SCHITTENHELM, M., Nucl. Fusion 37 (1997) 1255.

[37] KERNER, W., J. Comput. Phys 142 (1998) 271.

[38] BREIZMAN, B. N. et al., Phys. Plasmas 12 (2005) 112506.

[39] VAN-ZEELAND, M. A. et al., Phys. Rev. Lett. 97 (2006) 135001. 